\title{
Research on Electric Shock Prevention and First Aid for Electric Vehicles
}

\author{
Guo Hao \\ Tianjin University of Technology and Education \\ School of Automotive And Transportation \\ Tianjin, China
}

\author{
LingxiaoYang \\ Tianjin University of Technology and Education \\ School of Automotive And Transportation \\ Tianjin, China
}

\begin{abstract}
This paper analyzes the harm of electric current to human body and the person electric shock hazard may occur during the process of using electric vehicles, puts forward measures to prevent people from electric shock, and introduces the correct method of On-the-spot rescue in case of the electric shock accidents.
\end{abstract}

\section{Keywords-Electric vehicles; electrical safety; first aid}

\section{INTRODUCTION}

The traction battery supplies power to the drive motor, and the motor drives the wheels. The entire drive system, it includes traction battery, the converter, the drive motor, the transmission and the power cable. Additional auxiliary equipment also includes electric air conditioning compressor. These high-voltage components work at a voltage up to be $200-600 v$. AC voltage greater than $25 \mathrm{~V}$, DC voltage more than $60 \mathrm{~V}$ will pose a threat to human life. Therefore, once the electric vehicle caused a person to electric shock; the person is very dangerous. In the design of the electric vehicle, the direct protection and indirect protection should be adopted to reduce the probability of electric shock and the harm to the human body. [1] However, some uncertainties (e.g. Aging and corroded power cables, damaged power cable insulation, and Incorrect Operation by Maintenance Staff, etc.) may pose a threat to the lives of people during normal operating conditions and maintenance. The paper is organized as follows. Detailed theoretical analysis is reported for Electrical safety of electric vehicles in the next section. Preventive measures of the electric shock accidents from different aspects in the subsequent section. This is followed by the First aid method of the electric shock accidents. The conclusions are given in the last section.

\section{THE OCCURRENCE OF THE ELECTRIC SHOCK ACCIDENTS}

\section{A. Electrical Hazards of High-voltage Systems in Electric Vehicles}

Electric currents through the human body can cause electric shocks, breath and heartbeat interruptions, burns and permanent bodily injury and even death. In the IEC 60479-1, The relationship between the current flowing through the human body and the duration of the current on human physiological effects is given, as shown in Figure 1.

In the $\mathrm{AC}-1$ area, the flow of current through the human body is $0-0.5 \mathrm{~mA}$ and does not adversely affect the human body. The AC-2 area, $0.5-2 \mathrm{~mA}$, you can feel the current; $3-5$ $\mathrm{mA}$, the body feels pain, but can still get rid of the electric shock conductor at this time; $10-20 \mathrm{~mA}$, the current will trigger muscle spasms. The human body cannot actively control the muscle, resulting in a prolonged electric shock time; in the AC-3 area, electric currents can cause shock personnel to breathe difficult and arrhythmia; in the AC-4 area, electrical currents can cause cardiac fibrillation, cardiac arrest and stop breathing, risking life during this time. [2]. The different consequences depend on the voltage of the contact position of the electric shock, the flow of current through the human body and the current frequency. Usually, the most dangerous is that the current path is from hand through the heart to foot.

AC power flow through the body will ca use the risk of arrhythmia. Lower frequency of AC, the more damage to the human body. Direct current mainly causes chemical reaction inside a human body in the electric shock accident.

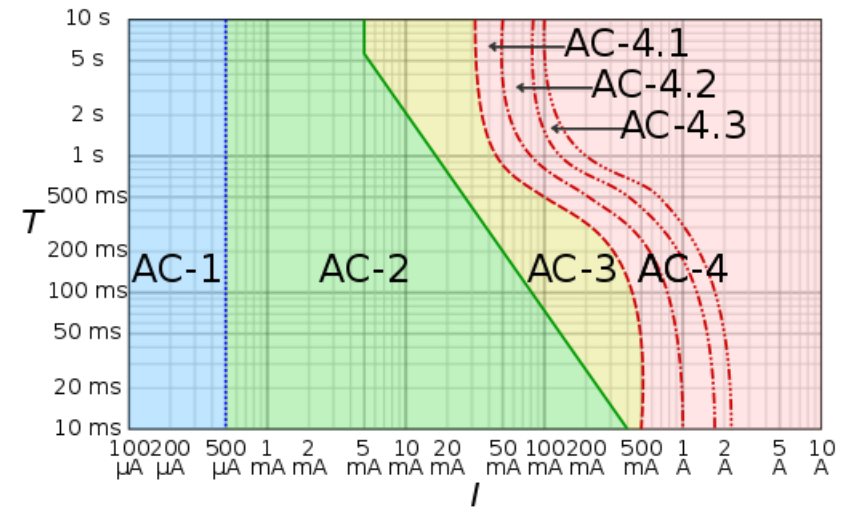

Fig. 1. The relationship between current and current duration of the human body

The human body resistance will be different because of sex, individual difference or skin's wet and dry degree and so on, in which the skin is dry, the human body resistance can be as high as $100,000 \Omega$, but in the case of sweat or wet skin, the body resistance has about $1,000 \Omega$. In theoretical studies, the selection of the human body resistance is approximately 1,000. Assuming that the high-voltage direct current on the electric vehicle is $300 \mathrm{~V}$, according to the Ohm's law, the current flow through the human body is $300 \mathrm{~mA}$, according to the figure 1 , if the current cannot be cut in a short period of time, Electric shockers may have a life threat. 


\section{B. Common Electric Shock Forms for Electric Vehicles}

The Electric shock means that there is an electric potential difference at both ends of the person's contact and the current flows through the body.In order to protect the safety of the electric vehicle users and maintenance personnel, the electric shock protection mainly includes direct and indirect contacts, in which direct contact refers to contact with parts normally operation, for example, the personnel directly contact with the high-voltage terminals. Indirect contact refers to a contact with an electrically charged device that is not electrically charged under normal conditions, but because of an insulating fault, such as a person with a shell of a leakage device. Direct contact protection can be regarded as the basic protection, which is the protection before the failure, for example, the insulating layer outside the high-voltage cable. Indirect contact protection is to minimize the damage to the human body caused by the failure of the basic protection, which belongs to the protection after the failure, such as the equipotential connection between each high-voltage component.

If the poles of the electric vehicle battery are isolated from the chassis, the failure of the basic insulation of exposed conductive components (i.e. metal enclosure of equipment) does not pose a threat to the safety of personnel in contact with the electric vehicle.

In the design of the electric vehicle, the traction battery and the high-voltage components adopt the IT topology as shown in the Figure 2, When the electric energy is transmitted, the high-voltage passes through the positive pole of the high-voltage power battery to each high-voltage element, and then goes back to the negative pole of the traction battery. During the whole transmission process, the current does not flow into the chassis, with the supply circuit isolated from the chassis. The equipotential bonding wire connects the metal enclosure of each high-voltage component to the chassis by welding or bolting ${ }^{[3]}$.

When the metal enclosure of high-voltage component is charged, the current flows through the equipotential bonding wire to the chassis, thus ensuring that current do not flow through the body when the person touches both the charged enclosure and the chassis. At this time, the system can still work. As shown in picture 2.

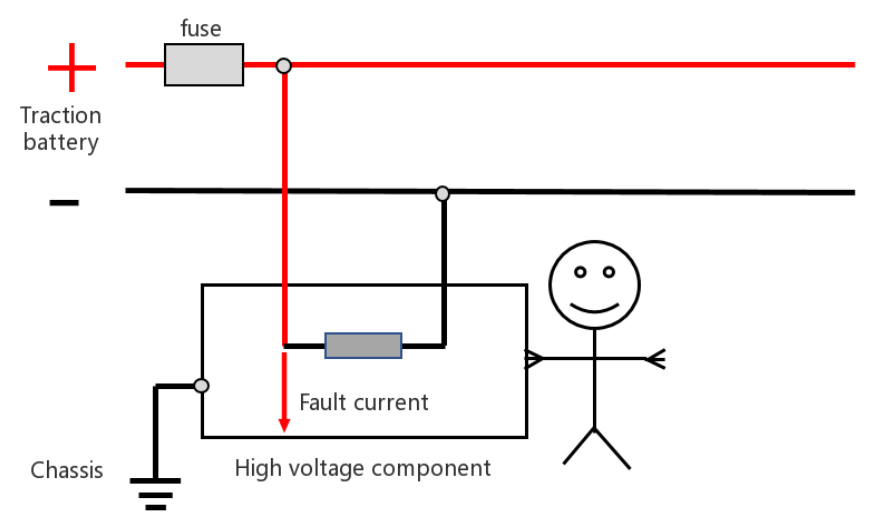

When two high-voltage components of the enclosure at the same time charged, even if the human body at the same time contact with the two charged enclosure, electric current forms current circuit in the equipotential bonding line, and will not flow through the human body, at this time, the entire system short-circuit, fuse blows in the high-voltage system, and the electric current is cut off. As shown in Figure 3.

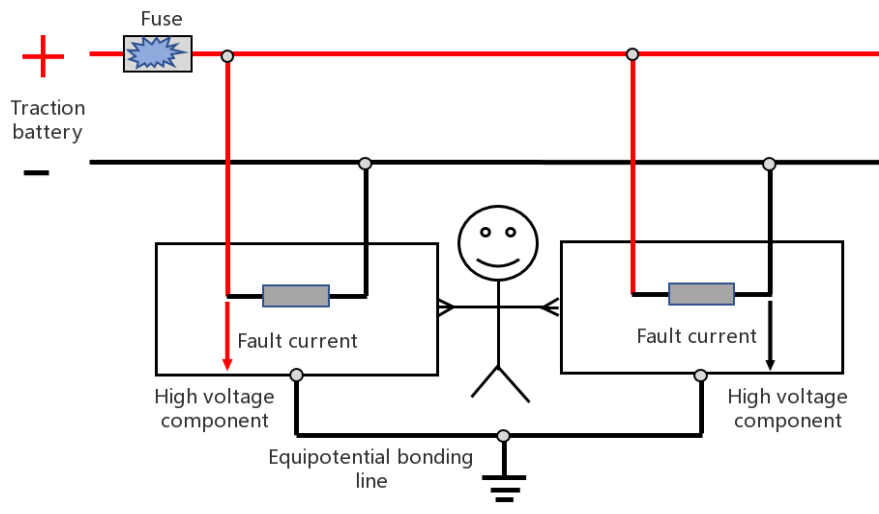

Fig. 3. The person does not get an electric shock when the second fault occurs and the equipotential bonding line is well connected.

During the use of electric vehicles, the equipotential bonding wire aging, wear, insulating layer rupture or cable rupture, and equipotential bonding point damp rust or connecting bolts loose or falling off by the body vibration and other uncertainties caused by poor insulation or failure, at the same time, high voltage components of internal damage or humidity will make the enclosure of High-voltage components charged, If the human body contact with two charged highvoltage component enclosure, the current cannot normally through the equipotential bonding wire to the chassis. There is a potential difference at both ends of the contact, the current flow through the human body, causing an electric shock accident. As shown in Figure 4.

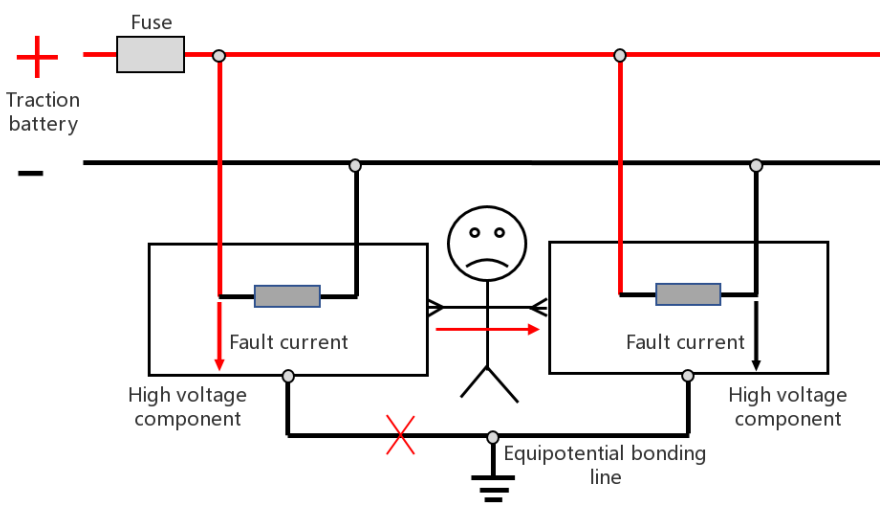

Fig. 4. When the equipotential bonding line fails, the person is at risk of electric shock

Fig. 2. The first failure occurs and the person is not electrocuted 


\section{THE PREVENTION OF THE ELECTRIC SHOCK ACCIDENTS}

Through the above analysis, once the electric shock accident, the life safety of electric shock personnel poses a serious threat, in the normal use of electric vehicles and maintenance process, should be as far as possible to reduce the probability of accidents, in addition to the electric vehicle high-voltage components to the personnel shock protection design, here are some measures to prevent electric shock accidents.

\section{A. Remote Monitoring Platforms}

In accordance with the provisions of Article 17 of the regulations regarding the admission of New Energy Automobile Manufacturers and products issued by the Ministry of Industry and Information Technology of the people's Republic of China in January 2017, the New Energy Automobile Manufacturers should establish a monitoring platform for the running safety condition of new energy automobile products. According to the agreement with new energy automobile product users, the running safety status of all new energy vehicle products that have been sold is monitored. Enterprise monitoring platform should be connected with local and national new energy vehicle monitoring platform docking ${ }^{[4]}$. The existing monitoring platform is mainly composed of electric vehicle terminal, enterprise Monitoring Platform server, enterprise monitoring Platform data processing platform, national or local monitoring platform, the main structure as shown in Figure 5. The remote monitoring platform analyzes the operating parameters of the high-voltage components uploaded by the vehicle terminal. Judges its working status, predicts the possible faults, and sends warning messages to the vehicle terminal to remind the driver.

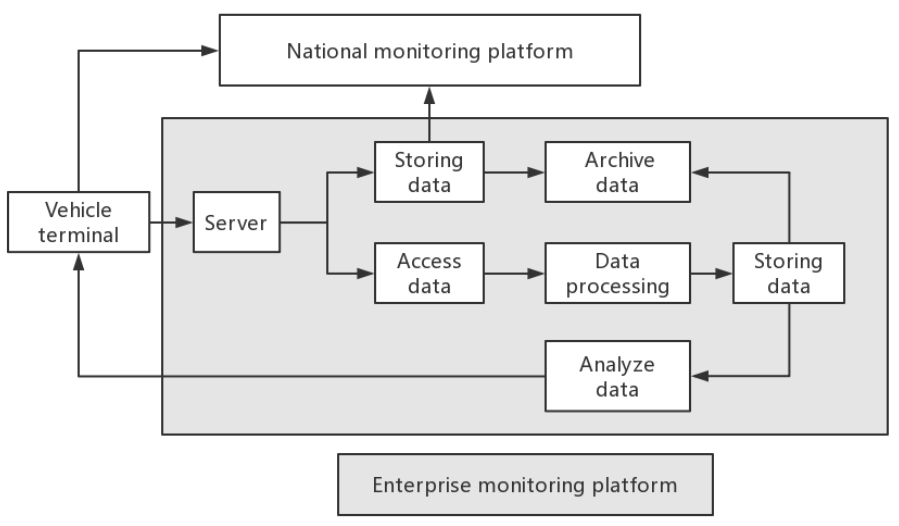

Fig. 5. Remote monitoring platform architecture for electric vehicles.

\section{B. System of Monitor the Insulation Fault}

The insulation resistance between the high-voltage components and the chassis is too small due to the bad operating environment during the use of electric vehicles, which will threaten the lives of drivers and passengers. However, not all high-voltage systems of electric vehicles include insulation monitoring systems. The high-voltage insulation monitoring systems of electric vehicles should continuously or intermittently monitor the insulation resistance of the entire high-voltage system, if a decrease as the insulation resistance is found, a warning should be issued, then disconnect the high-voltage system according to the running state of the vehicle or restrict the activation of the high-voltage system.

\section{High-voltage Operation Training}

In the maintenance of electric vehicles, the maintenance personnel must undergo complete high-voltage operation training and obtain the qualification certification of highvoltage maintenance personnel. At the same time, in the maintenance operation, maintenance personnel shall wear protective equipment and work strictly in accordance with the operational specifications. Professional maintenance personnel should remind drivers and passengers not to blindly touch the orange high-voltage power line or the high-voltage components connected to it on the electric car during use.

\section{ON-THE-SPOT RESCUE OF THE ELECTRIC SHOCK ACCIDENTS}

After When an electric shock happens, first of all, keep calm, and when you are in direct contact to a person who is not separated from the conductor, you will also be electrocuted. Therefore, according to the correct rescue process, the electroshock personnel can be rescued in the shortest time, and the safety of the rescuers can be guaranteed. The correct On-the-spot rescue process is shown in figure 6.

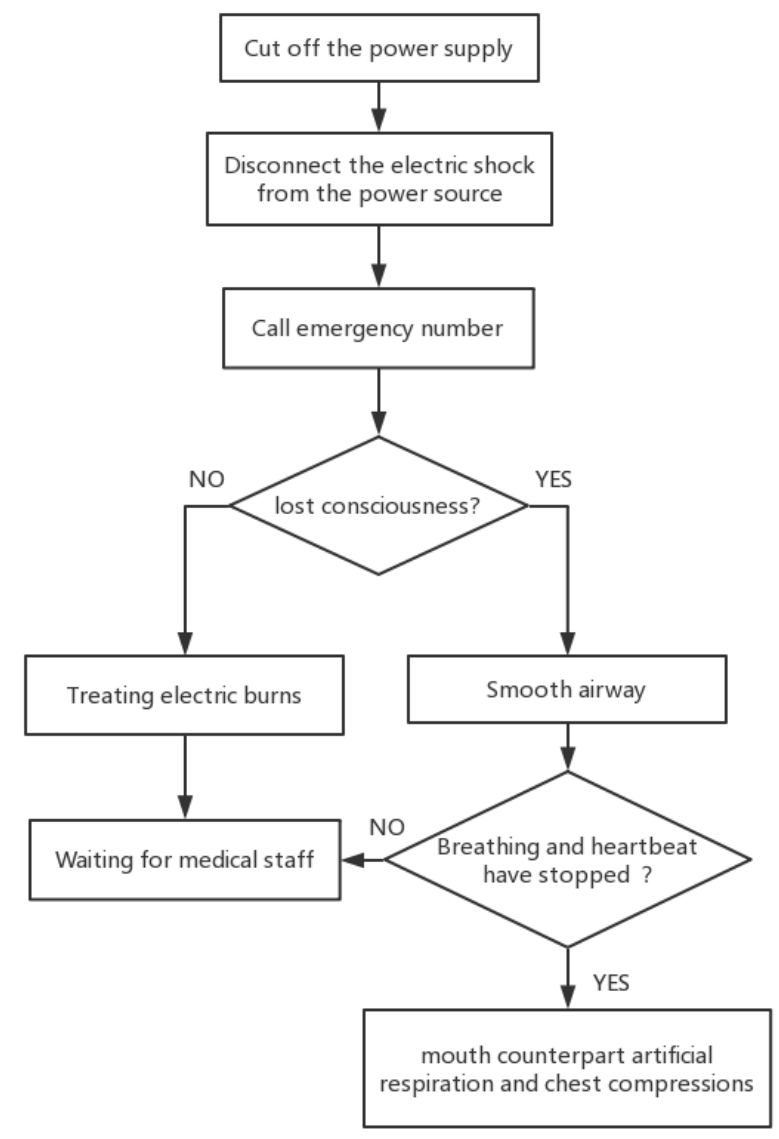

Fig. 6. On-the-spot rescue process of the electric shock accidents 


\section{CONCLUSION}

This paper introduces the possible electrical hazards and the form of electric shock that may exist in the use of electric vehicles and expounds how to prevent electric shock accidents in the design of electric vehicles and use of electric vehicles. If people are electrocuted and how to correctly rescue, this paper puts forward some guidance.

\section{REFERENCES}

[1] Freschi, Fabio , M. Mitolo , and R. Tommasini . "Electrical safety of electric vehicles. " Industrial \& Commercial Power Systems Technical Conference IEEE, 2017.
[2] IEC TS 60479-1: "Effects of current on human beings and livestock. Part 1: General aspects." 4th Ed., 2005.

[3] Zhao Jiawang, Liu Guibin, Zhou Rong. "Research on the protection of electric shock for electric vehicles, "Automotive Applied Technology, pp. $22-24,2016$

[4] Ministry of Industry and Information Technology of the People's Republic of China, "New energy vehicle production enterprises and product access management regulations." 2017: 1-17.

[5] ISO 6469-2:2009, "Electric road vehicles - Safety specifications - Part 2: Vehicle operational safety means and protection against failures"

[6] ISO 6469-3:2011, "Electrically propelled road vehicles. Safety specifications -Part 3: Protection of persons against electric shock." 